\title{
A Systematic Review on Long Term Effects of Weight Loss Diet on Body Weight and Lipid Profile: Findings from Randomized Controlled Trials
}

\author{
Nurul Umirah Abdul Manaf, Aryati Ahmad", Noor Aini Mohd Yusoff \\ School of Nutrition and Dietetics, Faculty of Health Sciences, Universiti Sultan Zainal Abidin, \\ 21300 Kuala Nerus, Terengganu, Malaysia
}

Copyright $(\mathrm{C} 2018$ by authors, all rights reserved. Authors agree that this article remains permanently open access under the terms of the Creative Commons Attribution License 4.0 International License

\begin{abstract}
Various diets with different macronutrient compositions have been designed and practiced to achieve desirable weight loss. However, evidence on the benefits and risks of these diets over long- term duration on weight loss and fasting serum lipid is still scarce with different opinions and perspectives. A systematic literature search for relevant eligible trials was conducted on randomized controlled trials (RCTs) with parallel design that examined the effects of weight loss diet on body weight and fasting serum lipid, in overweight or obese adults aged 18 to 50 years old; focusing on calorie restriction with specific macronutrient distribution. Nine trials with a total of 1016 individuals fulfilled inclusion criteria. After 12 months, low carbohydrate diet (LC) showed significant increment in body weight (weighted mean difference, $1.22 \mathrm{~kg}$ : $95 \%$ confidence interval (CI), 0.17 to $2.28, P=0.02$ ) and triglyceride (TG) value (weighted mean difference, $0.11 \mathrm{mmol} / \mathrm{L}, 95 \% \mathrm{CI}: 0.02,0.19, P=0.01)$. Low-density lipoprotein cholesterol (LDL-C) and high-density lipoprotein (HDL-C) did not significantly change after both diets. In conclusion, the long term use of LC should be reconsidered due to unfavorable effect of LC on weight changes and TG. Nevertheless, more long term studies are warranted to confirm the efficacy and safety of these weight loss diets.
\end{abstract}

Keywords Weight-reducing Diet, Obesity, Dietary Intervention, Macronutrient Distribution

\section{Introduction}

Obesity prevalence has increased by more than two-fold since 1980 . In 2014, more than 1.9 billion adults aged18 years and older, were overweight. Of these, over 600 million were obese [1]. In Malaysia, between 1996 and
2011, the prevalence of both overweight and obesity among adults aged $\geq 18$ years old had increased tremendously [2]. The prevalence for overweight was $16.6 \%, 29.1 \%$ and $29.4 \%$ in 1996, 2006 and 2011 respectively [2]. As for obesity, the increment was from $4.4 \%, 14 \%$ and $15.1 \%$ in 1996,2006 and 2011 respectively [2]. The major determinants of obesity are complex, but clearly involved new or altered interactions with the environment, particularly related to food supply, eating behaviours, family-work culture and practices, socio-economic status, urban design and public policy [3, 4]. Obesity has been shown to be associated with a broad range of health issues ranging from specific diseases such as type- 2 diabetes and hypertension, impaired quality of life, psychosocial disturbance, and limited access to quality healthcare [5]. The link between obesity and cardiovascular disease (CVD) has been established, in which obesity predisposes a person to a number of cardiovascular risk factors such as impaired glucose tolerance and type 2 diabetes, hypertension, dyslipidemia and sleep apnea. The possible factors that relate these two components have been discussed in Dixon, J.B (2010) [5].

Dietary intake is one of the important factors that involve in the primary prevention of obesity and/or its associated unfavorable health consequences. Low carbohydrate diet (LC) has become a popular alternative to conventional low fat diet (LF) for weight loss. Numerous studies have been conducted to compare the effectiveness of both diets on weight loss and lipid profile with inconclusive results. LC was found to be more effective for weight loss and cardiovascular risk factor reduction than LF [6-8] whilst LF showed significant improvement in total cholesterol (TC) and low- density lipoprotein (LDL) in other study [9]. In contrast, both LC and LF may be equally ineffective as the average amount of weight loss on both diets when practiced beyond one year has been modest and some also showed negligible results [10]. 
Nonetheless, weight loss can be successfully achieved independent of macronutrient composition when coupled with behavioral treatment, exercise and long term adherence to the diet $[11,12]$.

In order to ensure adequate intake of essential nutrients and reducing risk of chronic disease, Institute of Medicine has set the Acceptable Macronutrient Distribution Range (AMDR) as following; Carbohydrate: $45-65 \%$, Protein: $10-35 \%$ and Fat: $20-35 \%$ as a reference for healthy population [6]. Despite this, there were many contradictory guidelines for weight management, dietary treatment of obesity, and prevention of CVD and their corresponding effects on CVD risk factors with respect to macronutrient distribution alteration. The debate regarding which diet is superior to the others for obesity treatment has become intense especially on the effects of diet on weight loss and lipid profile in long term. Our objective was to examine the efficacy of weight-loss intervention diets with different macronutrient composition in promoting weight loss and improving lipid profile with notable focus on sustained weight loss at $\geq 12$ months.

\section{Materials and Methods}

Systematic search was done through Medline, Cochrane Central Register of Controlled Trials and Scopus databases, using the query 'weight loss diet OR reducing diet' AND 'weight reduction OR weight loss' AND "fasting serum lipid OR HDL OR LDL OR triglyceride OR total cholesterol". The search was conducted to identify randomized controlled trials that analyzed the effects of weight loss diets on body weight changes and fasting serum lipids published between January 2005 and June 2015. Additional articles were selected from reference lists of included studies, relevant reviews and previous metaanalyses.

\subsection{Inclusion Criteria}

The inclusion criteria for studies to be included in this review were:

1) randomized controlled trials with parallel design.

2) involved overweight or obese adults aged 18 to 50 years old.

3) RCTs that involved intervention on weight reducing diet only with or without control diet.

4) diet experimented in the studies focusing on calorie restriction with specific macronutrient distribution.

5) articles published between January 2005 and June 2015.

6) articles in English.

7) studies with intervention duration of $\geq 12$ months.

8) studies that reported the measures of weight loss in absolute change of weight loss, percentage of weight loss, and body mass index changes relative to baseline with body weight measured using validated and calibrated tools on fasting serum lipid with absolute changes in triglyceride (TG), high-density lipoprotein cholesterol (HDL-C), low-density lipoprotein cholesterol (LDL-C), total cholesterol (TC) pre and post intervention.

\subsection{Exclusion Criteria}

The studies were excluded from this review if 1) studies that involved subjects with obesity comorbidities (such as diabetes mellitus, cancer, epilepsy, hypertension) or altered endocrinological state (such as pregnancy or menopause); 2) studies that were confounded with factors that may affect weight loss such as medication/ supplements used and interventions that combined diet with exercise; 3) studies that involved children and elder; 4) incomplete review articles or abstract papers or published articles.

\subsection{Data Extraction and Analysis}

Data on study characteristics, study population, demographic and clinical characteristics and use of any co-interventions (e.g.: exercise, nutritional counselling, supplement/meal replacement used) were extracted using standardized form. Nutritional counselling was defined as any additional, clearly specified individual or group meeting with registered dietitian, nutritionists or other healthcare professionals or research staff, with training or experience in obesity management. Outcome data were extracted for the longest follow up time available. In studies where authors used multiple statistical methods, we extracted the results of their primary analysis. Our primary end points were sustained body weight loss (mean change final - baseline) while secondary end points were mean change in TC, TG, LDL-C and HDL-C over the study duration. 


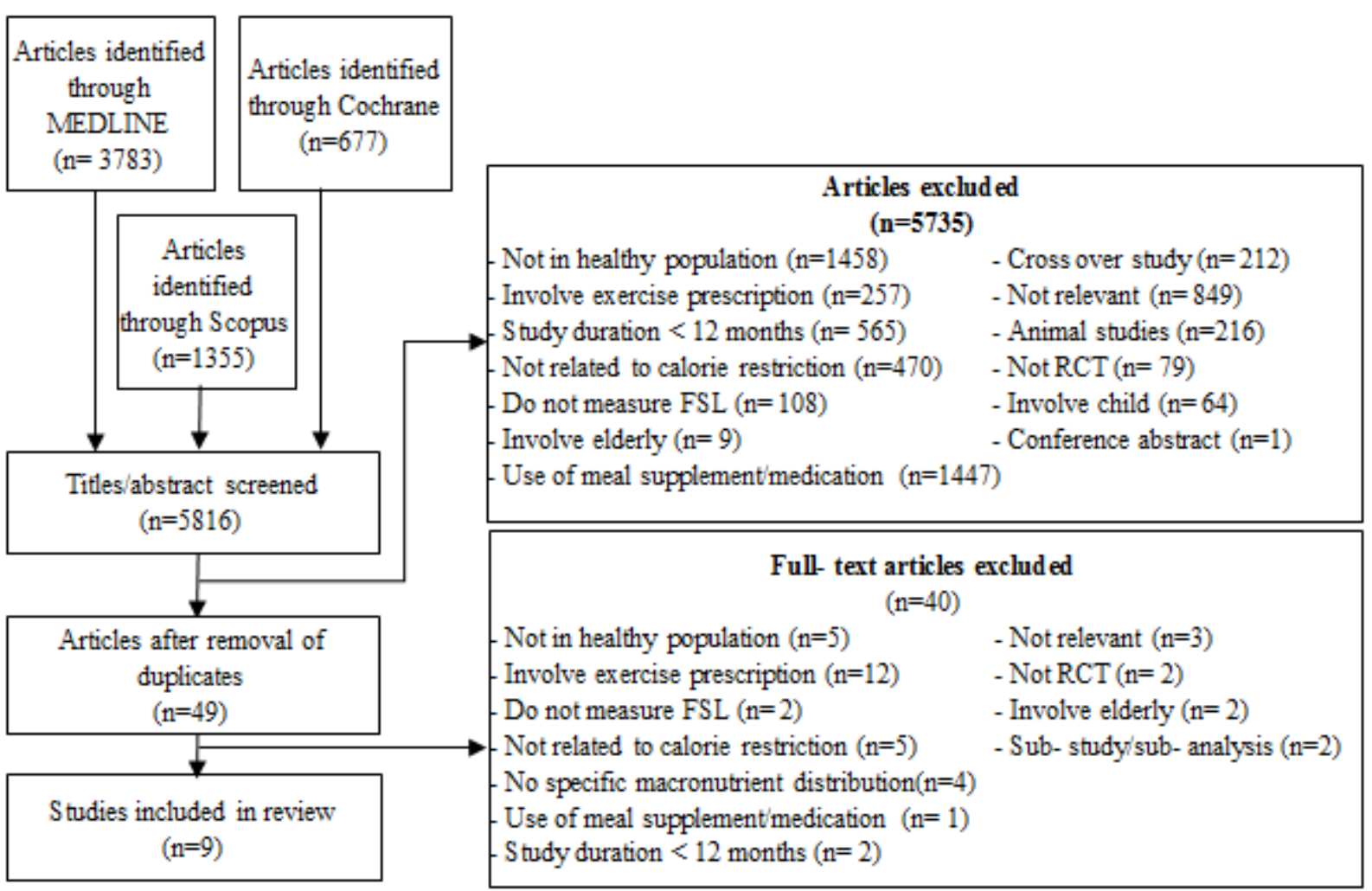

Figure 1. Study-flow diagram showing the number of studies screened, assessed for eligibility, and included in the review. RCT, randomized controlled trial; FSL, fasting serum lipid

\subsection{Statistical Analysis}

A meta-analysis was undertaken for each dietary-intervention subgroup when appropriate ( 8 of 9 trials) using Revman 5.3 software (Cochrane Information Management System). The heterogeneity was calculated using Cochrane's Q (Chi2) statistic and the inconsistency was measured (I2) across trials. The method used in the meta-analysis (random- or fixed-models) was determined by the degree of heterogeneity. Sensitivity analyses were conducted to explore heterogeneity.

\section{Results}

Initial search yielded 5816 potentially relevant articles (Figure 1). After screening the titles and abstracts as well as removal of duplicates between databases, 49 studies were retrieved for full- text article. Nine studies met eligibility criteria and included in this review, with data from 1016 trial participants (511 participants from low carbohydrate diet (LC) and 505 participants from low fat diet (LF)). Included trials were grouped into LC and LF based on their macronutrient composition for further analysis. The goal of carbohydrate composition for LC group was $<45 \%$ of total energy intake whilst fat consumption goal for LF group was $<30 \%$ of energy intake. Majority of the trials in LF group showed higher completion rate at the end of the study as compared to LC group except for one trial [13] which showed the vice versa. Out of nine trials, only five trials [13-17] fully described the reasons for losses to follow up.

Studies were excluded from the meta-analysis but included in the results section (Table 1) if data were not available [18]. The eight studies included in the metaanalysis were RCTs with dietary interventions ranged from 12 months $[13-17,19,20]$ to 24 months [11]. The studies included in this review assessed the effects of only weight reducing diet interventions with specific macronutrient distribution. Table 1 shows the characteristics of the nine trials; whilst Table 2 summarizes the participant's characteristics in each trial. Healthy overweight or obese individuals, whose mean age ranged from 27 to 52 years (Table 2) were included in this review.

All participants in the trials included in this review were free- living individuals who bought and/or prepared their own food except for one trial [9] in which the food were provided for the first 24 weeks of study duration. Changes in body weight are shown in Table 3 and lipid values in Table 4, 5, 6 and 7. 
Table 1. General Characteristics of Trials

\begin{tabular}{|c|c|c|}
\hline Study & Inclusion criteria & Interventions \\
\hline J.B Keogh et al (2007) & $\begin{array}{l}\text { Age } 20-65 \text { years old, BMI } 27-40 \mathrm{~kg} / \mathrm{m}^{2} \\
\text { fasting serum insulin }>15 \mathrm{IU} / \mathrm{l}\end{array}$ & $\begin{array}{l}\text { Diet Composition: High monounsaturated fat diet }(30: 20: 50) \text { High protein } \\
\text { diet }(30: 40: 30) \text {. Total calorie prescription } 6000 \mathrm{~kJ}(1434 \mathrm{kcal}) \text { per day } \\
\end{array}$ \\
\hline TP Wycherley et al (2012) & $\begin{array}{c}\text { Males, Age } 20-65 \text { years old, BMI } \\
27-40 \mathrm{~kg} / \mathrm{m}^{2}\end{array}$ & $\begin{array}{l}\text { Diet Composition: High protein diet }(40: 35: 25), \text { High carbohydrate diet } \\
(58: 17: 25)\end{array}$ \\
\hline Ebbeling et al (2007) & Age $18-35$ years old, BMI $>30 \mathrm{~kg} / \mathrm{m}^{2}$ & Diet Composition: LC (40:25:35), LF (55:25:20) \\
\hline Das et al(2007) & BMI $25-30 \mathrm{~kg} / \mathrm{m}^{2}$ & $\begin{array}{l}\text { Diet Composition : LC (40:30:30), LF (60:20:20) Phase 1: } 7 \text { week baseline } \\
\text { period (subject asked to maintain stable weight and continue usual dietary } \\
\text { intake Phase 2: 24- week calorie restriction phase, food provided } 70 \% \text { of } \\
\text { individual baseline EER Phase 3: 24- week calorie restriction phase, same } \\
\text { regime as before but food not provided }\end{array}$ \\
\hline Frisch et al (2009) & Age $18-70$ years old, $\mathrm{BMI}>27 \mathrm{~kg} / \mathrm{m}^{2}$ & $\begin{array}{l}\text { Diet Composition: High carbohydrate diet }(55: 15: 30) \text {, LC }(40: 25: 35) \text {. All } \\
\text { participants advised to reduce calorie intake by at least } 500 \mathrm{kcal} .\end{array}$ \\
\hline Layman et al (2009) & BMI $>26 \mathrm{~kg} / \mathrm{m}^{2}$, body weight $<14 \mathrm{~kg}$ & $\begin{array}{l}\text { Diet composition: LC (40:30:30), high carbohydrate diet }(55: 15: 30) \text {. Calorie } \\
\text { prescription were } 1700 \mathrm{kcal} / \mathrm{day} \text { for female and } 1900 \mathrm{kcal} / \mathrm{day} \text { for male }\end{array}$ \\
\hline J.B Keogh et al (2007) & Age $20-65$ years old, BMI $27-40 \mathrm{~kg} / \mathrm{m}^{2}$ & $\begin{array}{l}\text { Diet Composition: LC (33:40:27), High carbohydrate diet }(60: 20: 20) \text {.Total } \\
\text { calorie prescription } 6000 \mathrm{~kJ}(1434 \mathrm{kcal}) \text { per day }\end{array}$ \\
\hline \multicolumn{3}{|l|}{ OTHER DIETS } \\
\hline L. Azadbakht et al (2007) & $\begin{array}{l}\text { Overweight and obese men and women, not } \\
\text { participated in weight- reduction programs } \\
\text { during previous } 6 \text { months and maintained } \\
\text { stable weight }( \pm \mathrm{kg})\end{array}$ & $\begin{array}{l}\text { Diet Composition: LF }(65: 15: 20) \text {, moderate fat diet }(55: 15: 30) \text { Total calorie } \\
\text { prescription: minus } 500 \mathrm{kcal} \text { below energy needs based on body weight }\end{array}$ \\
\hline
\end{tabular}

Table 2. Baseline Participants Characteristics

\begin{tabular}{|c|c|c|c|c|c|c|c|}
\hline Study & Diet & $\mathrm{N}$ & $\begin{array}{c}\text { Age, } \\
\text { Mean(SD), y }\end{array}$ & $\begin{array}{c}\text { Sex, } \\
\text { No. }(\%), M\end{array}$ & $\begin{array}{c}\text { BMI, } \\
\text { Mean (SD) }\end{array}$ & Follow-up, mo & $\begin{array}{c}\text { Completion Rate at The End of } \\
\text { Trial, }(\%)\end{array}$ \\
\hline \multicolumn{8}{|c|}{ LC VS LF } \\
\hline \multirow[t]{2}{*}{ J.B Keogh et al (2007) } & LF & 19 & $48 \pm 13$ & NA & $34 \pm 4$ & 12 & 53 \\
\hline & LC & 19 & $52 \pm 8$ & NA & $3 \pm 4$ & & 51 \\
\hline \multirow[t]{2}{*}{ TP Wycherley et al (2012) } & LF & 58 & $51.3 \pm 9.4$ & \multirow{2}{*}{ NA } & \multirow{2}{*}{ NA } & 15 & 57 \\
\hline & LC & 62 & $50.2 \pm 9.3$ & & & & 56 \\
\hline \multirow[t]{2}{*}{ Ebbeling et al (2007) } & LF & 37 & $26.9 \pm 4.2$ & $8(22)$ & \multirow{2}{*}{ NA } & 18 & 78 \\
\hline & LC & 36 & $28.2 \pm 3.8$ & $7(19)$ & & & 64 \\
\hline \multirow[t]{2}{*}{ Das et al(2007) } & LF & 17 & $34 \pm 5$ & $4(24)$ & $27.5 \pm 1.6$ & 12 & 88 \\
\hline & LC & 17 & $35 \pm 6$ & $4(24)$ & $27.6 \pm 1.2$ & & 82 \\
\hline \multirow[t]{2}{*}{ Frisch et al (2009) } & LF & 100 & $47 \pm 10.8$ & $24(24)$ & $33.8 \pm 4.8$ & 12 & 80 \\
\hline & LC & 100 & $47 \pm 10.3$ & $38(38)$ & $33.5 \pm 3.9$ & & 85 \\
\hline \multirow[t]{2}{*}{ Layman et al (2009) } & LF & 64 & $45.2 \pm 9.6$ & $28(44)$ & $32.2 \pm 4$ & 12 & 64 \\
\hline & LC & 66 & $46.0 \pm 8$ & $31(47)$ & $32.7 \pm 4$ & & 45 \\
\hline \multirow[t]{2}{*}{ J.B Kogh et al (2007) } & LF & 12 & $46.9 \pm 5.5$ & NA & $33.2 \pm 2.8$ & 12 & NA \\
\hline & LC & 13 & $50.1 \pm 5.0$ & & $32.6 \pm 3.6$ & & \\
\hline \multirow[t]{2}{*}{ Foster et al (2010) } & LF & 154 & $44.9 \pm 10.2$ & $49(32)$ & $36.1 \pm 3.5$ & 24 & 68 \\
\hline & LC & 153 & $46.2 \pm 9.2$ & $50(33)$ & $36.1 \pm 3.6$ & & 58 \\
\hline \multicolumn{8}{|l|}{ OTHER DIETS } \\
\hline \multirow[t]{2}{*}{ L. Azadbakht et al (2007) } & LF & 44 & $46 \pm 6$ & $11(25)$ & $29.2 \pm 11$ & 14 & 88 \\
\hline & Moderate fat diet & 45 & $45 \pm 5$ & $14(32)$ & $29.0 \pm 10$ & & 90 \\
\hline
\end{tabular}

All values are mean \pm SD or mean $(95 \% \mathrm{CI})$ unless otherwise indicated.

Abbreviations: BMI, body mass index (calculated as weight in kilograms divided by the square of height in meters); LC, low carbohydrate diet; LF, low fat diet; NA, not available

\subsection{Weight Loss}

Out of eight trials included, only five trials $[11,13,16$, $17,19]$ provided mean changes of body weight from baseline. Within trials with study duration range of 12- 18 months $[11,13,16,17,19]$, average weight loss was greater after LC as compared to LF (range of mean changes
-5.8 to $-12.3 \mathrm{~kg}$ for LC) except for one trial [17] that showed greater weight loss in LF group. Based on the meta-analysis, overall, significant increase in body weight was associated with LC (weighted mean difference, $1.22 \mathrm{~kg}$, $95 \%$ CI: $0.17,2.28, P=0.02$ ). There was no significant heterogeneity in the net changes in body weight $\left(I^{2}=0 \%\right.$, $P=0.72$ ) (Figure 2). 
Table 3. Changes in Body Weight in Individuals Trials Comparing LC vs LF

\begin{tabular}{|c|c|c|c|c|c|c|c|}
\hline \multirow{2}{*}{ Trial } & \multirow{2}{*}{ F/up,mo } & \multicolumn{7}{|c|}{ Body weight, Mean (SD), kg } \\
\cline { 3 - 8 } & & \multicolumn{2}{|c|}{ Baseline } & \multicolumn{4}{c|}{ Changes } \\
\cline { 3 - 8 } & & LC & LF & LC & $\%$ & LF & $\%$ \\
\hline J.B Keogh et al (2007)* & 12 & $93 \pm 12$ & $99 \pm 15$ & $-7.6 \pm 8.1^{*}$ & -8.2 & $-4.8 \pm 6.6^{*}$ & -4.8 \\
\hline TP Wycherley et al (2012) & 15 & $106.0 \pm 12.9$ & $101.6 \pm 14.9$ & $-12.3 \pm 8.0^{*}$ & -11.6 & $-10.9 \pm 8.6^{*}$ & -10.7 \\
\hline Ebbeling et al (2007) & 18 & $103.5 \pm 17.3$ & $103.3 \pm 15.1$ & NA & NA & NA & NA \\
\hline Das et al(2007)1 & 12 & $78.0 \pm 9.3^{*}$ & $78.5 \pm 12.3 *$ & NA & $-7.8 \pm 5.0^{*}$ & NA & $-8.0 \pm 4.1^{*}$ \\
\hline Frisch et al (2009) & 12 & $100.3 \pm 15.9$ & $98.8 \pm 16.9$ & $-5.8 \pm 6.1$ & -5.8 & $-4.3 \pm 5.1$ & -4.4 \\
\hline Layman et al (2009) & 12 & $91.7 \pm 16$ & $93.8 \pm 13$ & $-10.4 \pm 7.7$ & -9.0 & $-8.4 \pm 4.9$ & -11.3 \\
\hline J.B Keogh et al (2007) & 12 & $91.5 \pm 14.8$ & $97.6 \pm 8.3$ & NA & NA & NA & NA \\
\hline Foster et al (2010) & 12 & $103.3 \pm 15.5$ & $103.5 \pm 14.4$ & $-10.9 \pm 7.7$ & -10.6 & $-10.8 \pm 9.9$ & -10.4 \\
\hline Foster et al (2010) & 24 & $103.3 \pm 15.5$ & $103.5 \pm 14.4$ & $-6.3 \pm 10.8$ & -6.1 & $-7.4 \pm 11.0$ & -7.1 \\
\hline
\end{tabular}

Mean change $=-7.84 \mathrm{~kg}(\mathrm{LF})$ vs $-9.4 \mathrm{~kg}(\mathrm{LC}) . *$ Mean values significantly different as compared to baseline, 1 Study only reported percentage change from baseline, Abbreviations: BMI, body mass index (calculated as weight in kilograms divided by the square of height in meters); LC, low carbohydrate diet; LF, low fat diet; NA, not available

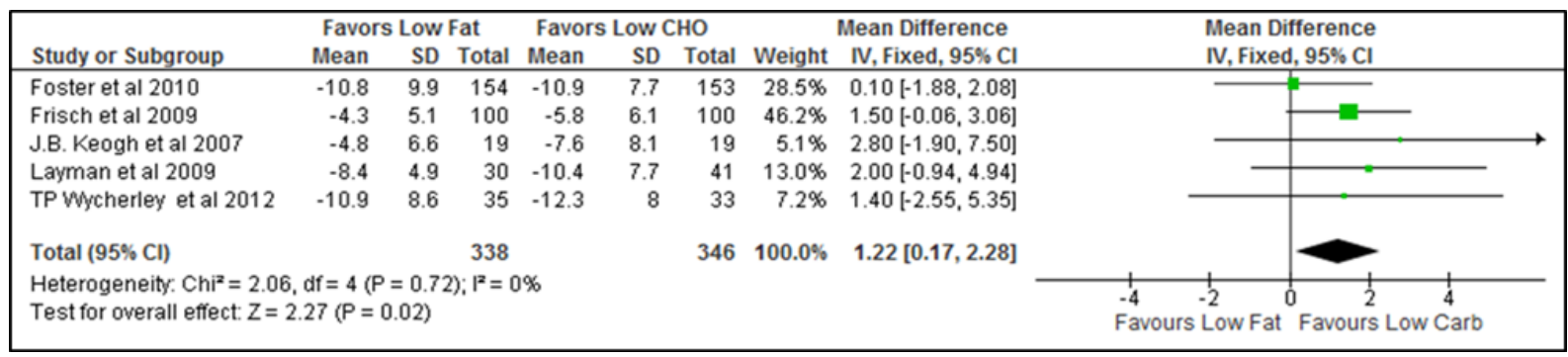

Figure 2. Weighted mean difference in weight loss. $\mathrm{CHO}$ indicate carbohydrates; $\mathrm{CI}$, confidence interval

\subsection{Changes in Lipids}

From the eight trials included in the review, only two trials $[13,16]$ provided data on mean changes of TC with a greater reduction in LF (mean change: $-0.42 \mathrm{mmol} / \mathrm{l}$ ) compared to LC (mean change: $-0.29 \mathrm{mmol} / 1$ ). However, the difference was not significant. Another trial by Frisch, S. et al (2009) [13] revealed increment in TC values in both diet groups after 12 months duration although the difference between groups was also not significant.

Only four trials provided data on mean changes of LDL-C from baseline. The difference between groups was not significant (weighted mean difference, $-0.01 \mathrm{mmol} / \mathrm{L}, 95 \% \mathrm{CI}:-0.17,-0.15, \mathrm{P}=0.87$ ). (Figure 3 ).

Four trials provided data on mean changes of HDL-C from baseline $[11,13,16,20]$. Of these, two trials $[13,20]$ had decreased HDL-C values from baseline while another two trials $[10,16]$ showed increment in HDL-C value. The difference in HDL-C was not significant between groups (weighted mean difference, $-0.05 \mathrm{mmol} / \mathrm{L}, 95 \% \mathrm{CI}:-0.11,0.00$, $\mathrm{P}=0.06$ ).

TG value from four available trials $[11,13,16,20]$ showed a decrease in value following 12-18 months of weight loss diets with the decrease was slightly higher in LC $[7,14,16]$ while one trial [16] showed the same decrement of TG for both groups. Based on the meta-analysis, significant increase in TG was associated with LC (weighted mean difference, $0.11 \mathrm{mmol} / \mathrm{L}, 95 \%$ CI: 0.02, 0.19, $\mathrm{P}=0.01$ ) (Figure 5).

Table 4. Changes in Total Cholesterol Values in Individuals Trials Comparing LC vs LF

\begin{tabular}{|c|c|c|c|c|c|c|c|}
\hline \multirow{3}{*}{ Trial } & \multirow{3}{*}{ Follow up, mo } & \multicolumn{6}{|c|}{ Total Cholesterol, Mean (SD), mmol/L } \\
\hline & & \multicolumn{2}{|c|}{ Baseline } & \multicolumn{4}{|c|}{ Changes } \\
\hline & & LC & LF & LC & $\%$ & LF & $\%$ \\
\hline J.B Keogh et al (2007) & 12 & $4.9 \pm 1.0$ & $5.6 \pm 1.2$ & NA & NA & NA & NA \\
\hline TP Wycherley et al (2012) & 15 & $5.1 \pm 1.0$ & $5.3 \pm 0.8$ & $-0.3 \pm 0.5^{*}$ & -5.7 & $-0.4 \pm 0.6^{*}$ & -7.9 \\
\hline Ebbeling et al (2007) & 18 & NA & NA & NA & NA & NA & NA \\
\hline Das et al(2007) 1 & 12 & $4.6 \pm 0.7$ & $4.4 \pm 0.7$ & NA & $-5.3 \pm 10.5^{*}$ & NA & $-4.2 \pm 9.3^{*}$ \\
\hline Frisch et al (2009) & 12 & $5.5 \pm 0.9$ & $3.6 \pm 0.9$ & $0.03 \pm 0.8$ & 0.5 & $0.1 \pm 0.6$ & 3.7 \\
\hline Layman et al (2009) & 12 & NA & NA & NA & NA & NA & NA \\
\hline J.B Keogh et al (2007) & 12 & $5.3 \pm 0.7$ & $5.7 \pm 1.0$ & NA & NA & NA & NA \\
\hline Foster et al (2010) & 12 & $4.9 \pm 0.8$ & $5.0 \pm 0.9$ & NA & NA & NA & NA \\
\hline Foster et al (2010) & 24 & $4.9 \pm 0.8$ & $5.0 \pm 0.9$ & NA & NA & NA & NA \\
\hline
\end{tabular}

Mean change $=-0.42 \mathrm{mmol} / 1$ (LF) vs. $-0.29 \mathrm{mmol} / 1$ (LC). *mean values significantly different as compared to baseline, 1 Study only reported percentage change from baseline, Abbreviations diet; LF, low fat diet; NA, not available: BMI, body mass index (calculated as weight in kilograms divided by the square of height in meters); LC, low carbohydrate 
Table 5. Changes in LDL-C Values in Individuals Trials Comparing LC vs LF

\begin{tabular}{|c|c|c|c|c|c|c|c|}
\hline \multirow{2}{*}{ Trial } & \multirow{2}{*}{ Follow up, mo } & \multicolumn{3}{|c|}{ LDL-C, Mean (SD), mmol/L } \\
\cline { 3 - 8 } & & \multicolumn{2}{|c|}{ Baseline } & \multicolumn{4}{|c|}{ Changes } \\
\cline { 3 - 8 } & & LC & LF & LC & $\%$ & LF & NA \\
\hline J.B Keogh et al (2007) & 12 & NA & NA & NA & NA & NA \\
\hline TP Wycherley et al (2012) & 15 & $3.2 \pm 0.9$ & $3.2 \pm 0.6$ & $-0.3 \pm 0.6^{*}$ & -8.4 & $-0.3 \pm 0.6^{*}$ & -9.7 \\
\hline Ebbeling et al (2007) & 18 & $2.6 \pm 0.9$ & $3.3 \pm 0.9$ & $-0.01 \pm 0.5$ & -0.4 & $-0.3 \pm 0.6$ & -8.3 \\
\hline Das et al(2007)1 & 12 & $2.8 \pm 0.6$ & $2.5 \pm 0.6$ & NA & $-7.0 \pm 17.5^{*}$ & NA & $7.1 \pm 11.3^{*}$ \\
\hline Frisch et al (2009) & 12 & $3.5 \pm 0.8$ & $3.6 \pm 0.9$ & $0.02 \pm 0.7$ & 0.6 & $0.1 \pm 0.6$ & 1.7 \\
\hline Layman et al (2009) & 12 & NA & NA & NA & NA & NA & NA \\
\hline J.B Keogh et al (2007) & 12 & $3.5 \pm 0.7$ & $3.8 \pm 1.0$ & NA & NA & NA & NA \\
\hline Foster et al (2010) & 12 & $3.1 \pm 0.7$ & $3.2 \pm 0.8$ & $-0.3 \pm 0.7$ & -8.0 & $-0.2 \pm 0.7$ & -6.9 \\
\hline Foster et al (2010) & 24 & $3.1 \pm 0.7$ & $3.2 \pm 0.8$ & $-0.1 \pm 0.7$ & -3.9 & $-0.2 \pm 0.6$ & -6.5 \\
\hline
\end{tabular}

Mean change $=-0.18 \mathrm{mmol} / 1$ (LF) vs. $-0.15 \mathrm{mmol} / 1$ (LC). *mean value significantly different as compared to baseline, 1 Study only reported percentage change from baseline, Abbreviations: BMI, body mass index (calculated as weight in kilograms divided by the square of height in meters); LC, low carbohydrate diet; LF, low fat diet; NA, not available

Table 6. Changes in HDL-C Values in Individuals Trials Comparing LC vs LF

\begin{tabular}{|c|c|c|c|c|c|c|c|}
\hline \multirow{2}{*}{ Trial } & \multirow{2}{*}{ Follow up, mo } & \multicolumn{3}{|c|}{ HDL-C, Mean (SD), mmol/L } \\
\cline { 3 - 8 } & & \multicolumn{2}{|c|}{ Baseline } & \multicolumn{4}{|c|}{ Changes } \\
\cline { 3 - 8 } & & LC & LF & LC & $\%$ & LF & NA \\
\hline J.B Keogh et al (2007) & 12 & $1.0 \pm 0.3$ & $1.1 \pm 0.3$ & NA & NA & NA \\
\hline TP Wycherley et al (2012 & 15 & $1.2 \pm 0.4$ & $1.3 \pm 0.4$ & $0.1 \pm 0.2 *$ & 10.6 & $0.1 \pm 0.2^{*}$ & 4.6 \\
\hline Ebbeling et al (2007) & 18 & $1.5 \pm 0.5$ & $1.4 \pm 0.3$ & $-0.1 \pm 0.2$ & -6.8 & $-0.2 \pm 0.2$ & -15.0 \\
\hline Das et al(2007)1 & 12 & $1.3 \pm 0.3$ & $1.4 \pm 0.2$ & NA & $11.9 \pm 10.2^{*}$ & NA & $13.3 \pm 16.2^{*}$ \\
\hline Frisch et al (2009) & 12 & $1.5 \pm 0.4$ & $1.5 \pm 0.4$ & $-0.02 \pm 0.2$ & -1.3 & $-0.03 \pm 0.2$ & -2.1 \\
\hline Layman et al (2009) & 12 & NA & NA & NA & NA & NA & NA \\
\hline J.B Keogh et al (2007) & 12 & $1.3 \pm 0.4$ & $1.3 \pm 0.4$ & NA & NA & NA & NA \\
\hline Foster et al (2010) & 12 & $1.2 \pm 0.4$ & $1.2 \pm 0.3$ & $0.2 \pm 0.3 * *$ & 17.5 & $0.1 \pm 0.2$ & 8.5 \\
\hline Foster et al (2010) & 24 & $1.2 \pm 0.4$ & $1.2 \pm 0.3$ & $0.2 \pm 0.3 * *$ & 16.7 & $0.1 \pm 0.2$ & 10.2 \\
\hline
\end{tabular}

Mean change $=-0.01 \mathrm{mmol} / \mathrm{l}(\mathrm{LF})$ vs. $0.05 \mathrm{mmol} / \mathrm{l}(\mathrm{LC})$. *mean values significantly different as compared to baseline, $* *$ Significant difference between groups 1 , Study only reported percentage change from baseline, Abbreviations: BMI, body mass index (calculated as weight in kilograms divided by the square of height in meters); LC, low carbohydrate diet; LF, low fat diet; NA, not available

Table 7. Changes in TG Values in Individuals Trials Comparing LC vs LF

\begin{tabular}{|c|c|c|c|c|c|c|c|}
\hline \multirow{2}{*}{ Trial } & \multirow{2}{*}{ Follow up, mo } & \multicolumn{3}{|c|}{ TG, Mean (SD), mmol/L } \\
\cline { 3 - 8 } & & \multicolumn{2}{|c|}{ Baseline } & \multicolumn{4}{|c|}{ Changes } \\
\cline { 3 - 8 } & & LC & LF & LC & $\%$ & LF & NA \\
\hline J.B Keogh et al (2007) & 12 & $2.3 \pm 1.0$ & $2.2 \pm 1.2$ & NA & NA & NA \\
\hline TP Wycherley et al (2012) & 15 & $1.5 \pm 0.5$ & $1.9 \pm 0.8$ & $-0.4 \pm 0.7$ & -23.5 & $-0.4 \pm 0.6$ & -23.8 \\
\hline Ebbeling et al (2007) & 18 & $1.3 \pm 1.1$ & $1.4 \pm 0.9$ & $-0.1 \pm 0.4$ & -7.9 & $-0.02 \pm 0.4$ & -1.4 \\
\hline Das et al(2007)1 & 12 & $1.1 \pm 0.4$ & $1.02 \pm 0.5$ & NA & $-15.2 \pm 24.8^{*}$ & NA & $16.5 \pm 29.9 *$ \\
\hline Frisch et al (2009) & 12 & $1.3 \pm 0.6$ & $1.4 \pm 0.7$ & $-0.1 \pm 0.5$ & -7.6 & $-0.04 \pm 0.5$ & -2.9 \\
\hline Layman et al (2009) & 12 & NA & NA & NA & NA & NA & NA \\
\hline J.B Keogh et al (2007) & 12 & $1.7 \pm 0.7$ & $1.4 \pm 0.4$ & NA & NA & NA & NA \\
\hline Foster et al (2010) & 12 & $1.3 \pm 0.6$ & $1.4 \pm 0.8$ & $-0.4 \pm 0.6$ & -28.1 & $-0.2 \pm 0.7$ & -14.2 \\
\hline Foster et al (2010) & 24 & $1.3 \pm 0.6$ & $1.4 \pm 0.8$ & $-0.14 \pm 0.8$ & -10.9 & $-0.2 \pm 0.8$ & -11.4 \\
\hline
\end{tabular}

Mean change $=-0.17 \mathrm{mmol} / 1$ (LF) vs. $-0.25 \mathrm{mmol} / 1(\mathrm{LC}) . *$ mean values significantly different as compared to baseline, $* *$ Significant difference between groups, 1 Study only reported percentage change from baseline, Abbreviations: TG, triglyceride; BMI, body mass index (calculated as weight in kilograms divided by the square of height in meters); LC, low carbohydrate diet; LF, low fat diet; NA, not available 


\begin{tabular}{|c|c|c|c|c|c|c|c|c|c|}
\hline \multirow[b]{2}{*}{ Study or Subgroup } & \multicolumn{3}{|c|}{ Favors Low Fat } & \multicolumn{3}{|c|}{ Favors Low CHO } & \multirow[b]{2}{*}{ Weight } & \multirow{2}{*}{$\begin{array}{l}\text { Mean Difference } \\
\text { IV, Random, } 95 \% \mathrm{Cl}\end{array}$} & \multirow{2}{*}{$\begin{array}{c}\text { Mean Difference } \\
\text { IV, Random, } 95 \% \mathrm{Cl}\end{array}$} \\
\hline & Mean & SD & Total & Mean & SD & Total & & & \\
\hline Ebbeling et al 2007 & -0.3 & 0.6 & 37 & -0.01 & 0.5 & 36 & $21.1 \%$ & $-0.29[-0.54,-0.04]$ & $\longrightarrow-$ \\
\hline Foster et al 2010 & -0.22 & 0.7 & 154 & -0.3 & 0.7 & 153 & $31.7 \%$ & $0.08[-0.08,0.24]$ & \\
\hline Frisch et al 2009 & 0.1 & 0.6 & 100 & 0.02 & 0.7 & 100 & $28.8 \%$ & $0.08[-0.10,0.26]$ & $f=-$ \\
\hline TP Wycherley et al 2012 & -0.3 & 0.6 & 35 & -0.3 & 0.6 & 33 & $18.4 \%$ & $0.00[-0.29,0.29]$ & \\
\hline Total $(95 \% \mathrm{Cl})$ & & & 326 & & & 322 & $100.0 \%$ & $-0.01[-0.17,0.15]$ & \\
\hline \multicolumn{9}{|c|}{$\begin{array}{l}\text { Heterogeneity: } \operatorname{Tau}^{2}=0.01 ; \mathrm{Chi}^{2}=6.74, \mathrm{df}=3(\mathrm{P}=0.08) ; \mathrm{I}^{2}=56 \% \\
\text { Test for overall effect: } Z=0.16(P=0.87)\end{array}$} & $\begin{array}{ccccc}1 & 1 & 1 & 1 \\
-1 & -0.5 & 0 & 0.5 & 1 \\
\text { Favours Low Fat } & \text { Favours Low } \mathrm{CHO}\end{array}$ \\
\hline
\end{tabular}

Figure 3. Weighted mean difference in LDL-C. CHO indicate carbohydrates; CI, confidence interval

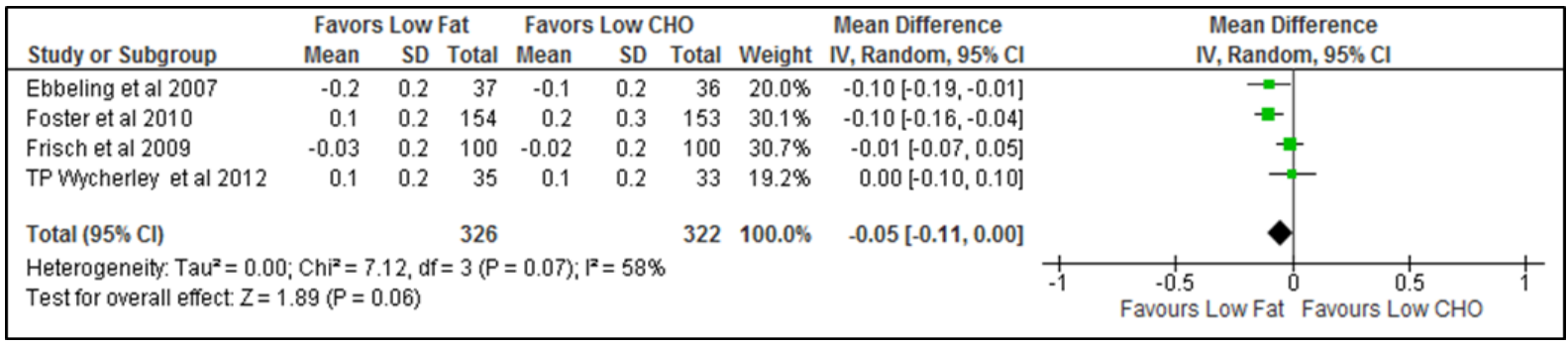

Figure 4. Weighted mean difference in HDL-C. CHO indicate carbohydrates; CI, confidence interval

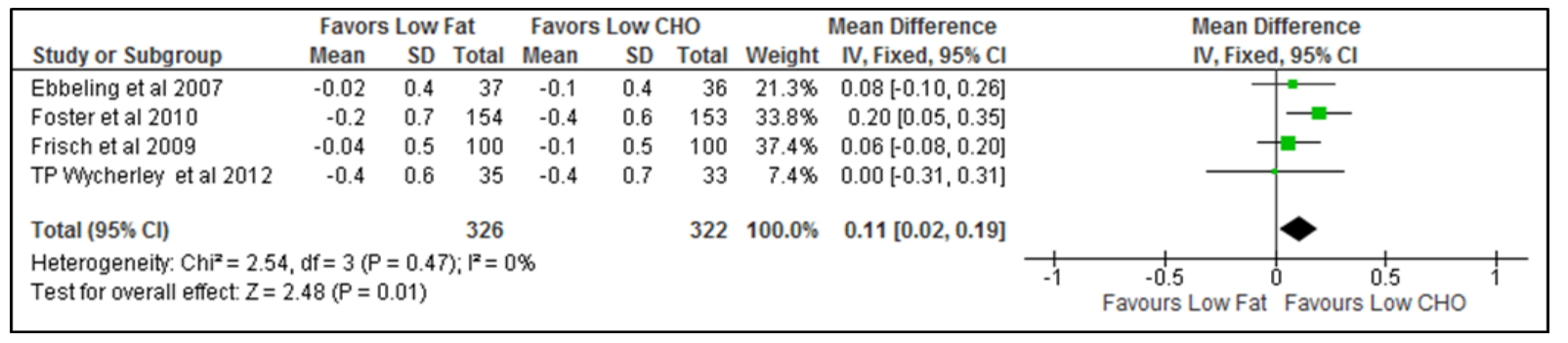

Figure 5. Weighted mean difference in TG. CHO indicate carbohydrates; CI, confidence interval

\section{Discussion}

This meta-analysis of randomized controlled trials comparing LF and LC diets based on their macronutrient composition found that $\mathrm{LC}$ was unfavorable for long term as it showed an increment in both body weight as well as TG value. There was no significant difference in the changes of LDL-C and HDL-C values for both diets. These findings questioned LC as an alternative approach for weight reduction for a long term use and should be reconsidered for practice. The small number of studies included in this review reflected that limited RCTs have been conducted regarding this topic, which focusing on effectiveness of the diet alone, independent of physical activity and pharmacologic approaches. This called for more intervention studies on this topic especially large scale study with longer duration of beyond 12 months.

Despite greater improvement of weight loss seen in LC in previous meta-analyses $[8,21]$, several studies were unable to reach conclusion regarding the effectiveness of $\mathrm{LC}$ on inducing weight loss in long term $[22,23]$. Both studies found that weight loss in LC is greater than of LF in 6 months, but the effect was no longer significant at 12 months $[22,23]$. Previous systematic review has noted that much of the weight loss achieved early during follow up of less than 6 months will be regained overtime [24], suggesting an attenuation of treatment effect at longer follow up. A study suggested that LC did not contribute to improvement in metabolic markers whilst the weight loss from LC simply resulted from reduced caloric intake due to increased satiety effect of protein [25]. There were differences in estimated magnitude of weight loss between particular studies and this review. The potential reasons for these differences were due to different composition of LC used. Bueno et al (2012) implemented LC with less than $50 \mathrm{~g}$ of carbohydrate intake per day while LC diet in Sackner-Bernstein et al (2015) was defined as total carbohydrate intake of $\leq 120 \mathrm{~g} /$ day $[8,21]$. On the other hand, our review used a relatively less stringent definition of daily carbohydrate intake of less than $45 \%$ from total energy intake (approximately equivalently to $168.75 \mathrm{~g} /$ day of carbohydrate in $1800 \mathrm{kcal} /$ day).

Use of LC as an alternative weight loss approach should be reconsidered as this review showed a significant increment in TG after LC. Elevated TG concentration has been identified as independent risk factors for cardiovascular disease [26]. Additionally, LC was not found to be superior to LF in terms of improvement in LDL-C and HDL-C. Contrary to this review, previous literature found a favorable effect of LC on lipid profiles [27]. However, the effects were only obvious in short term whilst long term effect was unknown [27]. Improvement in 
lipid profile was found to be closely related to weight loss [28]. With majority of the studies showed that the weight outcome declined over time, the changes in these lipid profile parameters cannot be ruled out. Nonetheless, no weight loss studies have been conducted with sufficient duration to study this effect [22].

The safety, efficacy and optimum level of carbohydrate restriction for $\mathrm{LC}$ has been questioned. $\mathrm{LC}$ was only found to be safe for practice over 6 months duration with lack of studies done to study the efficacy and safety of LC over long term [22]. Previous review reported a trend towards increased weight loss for diets with lower carbohydrate content: $-3.6 \mathrm{~kg}$ for $<60 \mathrm{~g}$ of carbohydrate intake daily versus $-2.1 \mathrm{~kg}$ for $>60 \mathrm{~g}$ daily [29]. However, the comparison was not vague as the studies included in the review were not isocaloric, thus the differences in weight loss magnitude may be contributed by reduction in energy intake rather than carbohydrate content. It is highly recommended that individuals who are trying to lose weight to practice a reduced calories diet with combination of physical activity, which has been scientifically approved for its effectiveness and maintenance of weight in long term [30].

This review had several limitations. First, losses to follow up were substantial, where there was an imbalance in completion rate among the intervention arms of the studies. Only $33 \%$ of the studies included in this review had completion rate of more than $80 \%$. Forty-four percent $(44 \%)$ of the included studies had completion rate of less than $70 \%$ (with minimum of $45 \%$ completion rate). Comparing between two diet groups, the dropout rate was higher in LC group, suggesting poor adherence to diet despite its beneficial outcomes. Secondly, the data available from the component of the studies included did not consistently disclose the quality of fat in the diets such as the proportion of saturated, monounsaturated fatty acid (MUF A) and polyunsaturated fatty acid (PUFA) except for two studies that stated the intake $<10 \%$ of saturated fat from the total fat intake [13] and 6\% from PUFA and 13\% from MUFA [14]. Although the diet goals had the same content of carbohydrate, protein and fat, the contents of fiber and micronutrients might be differed, which accounted for varieties of possible outcomes. Thus, the focus of dietary intervention should be shifted towards the type of foods in the diets instead of solely concerned on macronutrient composition [22].

There were also several strengths in this present review. The studies included were all randomized controlled trials; a gold standard for evaluating the effect of intervention and subjected to fewer biases than observational studies. Moreover, we only included studies with study duration of $\geq 12$ months to specifically examine the effects of these diets on long term basis as little is known about their effectiveness. However, the longest follow up duration in this review was 24 months [11], suggesting that there is a lack of trials being conducted for a longer duration. A guideline published by American College of Cardiology, American Heart Association and The Obesity Society [31] proposed that a trial should ideally have a duration of at least 24 months for management of overweight and obesity in adults with the target to improve adherence to dietary strategies. This review also focused on changes in weight loss and lipid profile following dietary intervention without any co-interventions such as physical activity and pharmacologic approach. This lends additional confidence to our conclusions when comparing the effectiveness of both LC and LF diet groups.

\section{Conclusions}

With the fundamental result from this meta- analysis, LC diet increased weight and TG in long term whilst the effects of both LC and LF diets on LDL-C and HDL-C were not significant. Looking on these outcomes, the use of LC as an alternative weight loss diet should be reconsidered due to unfavorable effects on weight changes and TG in a long term. Recommendation regarding which diet is better for clinical practice was inconclusive, calling for more long term clinical trials to further explore the effectiveness and safety of these diets. In addition, future studies should explore approaches to limit weight regain over time and achieve greater weight loss especially strategies in maintaining better adherence towards the diet. Nevertheless, weight loss can be successfully achieved independent of macronutrient composition. Individualized dietary modification with calorie restriction and healthy selection of food consumed may bring positive result when coupled with behavioral treatment, physical activity and long term adherence to the diet.

\section{Acknowledgements}

We would like to thank all team members who provided great assistance throughout completing this review.

\section{REFERENCES}

[1] WHO | Cardiovascular diseases (CVDs). WHOhttp:/www.who.int/mediacentre/factsheets/fs317/en/ (2016, accessed 9 March 2017).

[2] Institute for Public Health. National Health and Morbidity Survey 2015 (NHMS 2015). Vol. II: Non-Communicable Diseases, Risk Factors \& Other Health Problems. 2015. Epub ahead of print 2015. DOI: 10.1017/CBO9781107415324.004.

[3] Chan RSM, Woo J. Prevention of overweight and obesity: how effective is the current public health approach. Int $J$ Environ Res Public Health 2010; 7: 765-83. 
[4] Popkin BM, Gordon-Larsen P. The nutrition transition: worldwide obesity dynamics and their determinants. Int $J$ Obes 2004; 28: S2-S9.

[5] Dixon JB. The effect of obesity on health outcomes. Mol Cell Endocrinol 2010; 316: 104-108.

[6] Bazzano LA, Hu T, Reynolds K, et al. Effects of Low-Carbohydrate and Low-Fat Diets. Ann Intern Med 2014; 161: 309 .

[7] $\mathrm{Hu}$ T, Mills KT, Yao L, et al. Effects of low-carbohydrate diets versus low-fat diets on metabolic risk factors: A meta-analysis of randomized controlled clinical trials. Am J Epidemiol; 176. Epub ahead of print 2012. DOI: 10.1093/aje/kws264.

[8] Sackner-Bernstein J, Kanter D, Kaul S. Dietary Intervention for Overweight and Obese Adults: Comparison of Low-Carbohydrate and Low-Fat Diets. A Meta-Analysis. PLoS One 2015; 10: e0139817.

[9] Schwingshackl L, Hoffmann G. Long-term effects of low-fat diets either low or high in protein on cardiovascular and metabolic risk factors: a systematic review and meta-analysis. NutrJ 2013; 12:48. doi: 12-48.

[10] Gardner CD. Tailoring dietary approaches for weight loss. Int J Obes Suppl 2012; 2: S11-S15.

[11] Foster GD, Wyatt HR, Hill JO, et al. Weight and metabolic outcomes after 2 years on a low-carbohydrate versus low-fat diet: A randomized trial. Ann Intern Med 2010; 153: 147157.

[12] $\mathrm{Hu} \mathrm{T}$, Yao $\mathrm{L}$, Reynolds $\mathrm{K}$, et al. Adherence to low-carbohydrate and low-fat diets in relation to weight loss and cardiovascular risk factors. Obes Sci Pract 2016; 2: 24 31.

[13] Frisch S, Zittermann A, Berthold HK, et al. A randomized controlled trial on the efficacy of carbohydrate-reduced or fat-reduced diets in patients attending a telemedically guided weight loss program. Cardiovasc Diabetol 2009; 8: 36.

[14] Keogh JB, Brinkworth GD, Clifton PM. Effects of weight loss on a low-carbohydrate diet on flow-mediated dilatation, adhesion molecules and adiponectin. Br $J$ Nutr 2007; 98: $852-859$

[15] Das SK, Gilhooly CH, Golden JK, et al. Long-term effects of 2 energy-restricted diets differing in glycemic load on dietary adherence, body composition, and metabolism in CALERIE: A 1-y randomized controlled trial. Am J Clin Nutr 2007; 85: 1023-1030.

[16] Wycherley TP, Brinkworth GD, Clifton PM, et al. Comparison of the effects of 52 weeks weight loss with either a high-protein or high-carbohydrate diet on body composition and cardiometabolic risk factors in overweight and obese males. Nutr Diabetes 2012; 2: e40.

[17] Layman DK, Evans EM, Erickson D, et al. A Moderate-Protein Diet Produces Sustained Weight Loss and Long-Term Changes in Body Composition and Blood Lipids in Obese Adults. J Nutr 2009; jn.108.099440.

[18] Azadbakht L, Mirmiran P, Esmaillzadeh A, et al. Better dietary adherence and weight maintenance achieved by a long-term moderate-fat diet. Br J Nutr 2007; 97: 399-404.

[19] Keogh JB, Luscombe-Marsh ND, Noakes M, et al. Long-term weight maintenance and cardiovascular risk factors are not different following weight loss on carbohydrate-restricted diets high in either monounsaturated fat or protein in obese hyperinsulinaemic men and women. Br J Nutr 2007; 97: 405-410.

[20] Ebbeling CB, Leidig MM, Feldman HA, et al. Effects of a low-glycemic load vs low-fat diet in obese young adults: A randomized trial. JAMA 2007; 297: 2092-20102.

[21] Bueno NB, Vieira De Melo IS, Lima De Oliveira S, et al Very-low-carbohydrate ketogenic diet v. low-fat diet for long-term weight loss: a meta-analysis of randomised controlled trials. Br J Nutr 2013; 110: 1178-1187.

[22] Astrup PA, Meinert Larsen DT, Harper A. Atkins and other low-carbohydrate diets: Hoax or an effective tool for weight loss? Lancet 2004; 364: 897-899.

[23] Nordmann AJ, Nordmann A, Briel M, et al. Effects of low-carbohydrate vs low-fat diets on weight loss and cardiovascular risk factors: a meta-analysis of randomized controlled trials. Arch Intern Med 2006; 166: 285-293.

[24] Atallah R, Filion KB, Wakil SM, et al. Long-Term Effects of 4 Popular Diets on Weight Loss and Cardiovascular Risk Factors. Circ Cardiovasc Qual Outcomes 2014; 7: 815 LP-827.

[25] Paoli A, Rubini A, Volek JS, et al. Beyond weight loss: a review of the therapeutic uses of very-low-carbohydrate (ketogenic) diets. Eur J Clin Nutr 2013; 67: 789-796.

[26] Jacobson TA, Miller M, Schaefer EJ. Hypertriglyceridemia and cardiovascular risk reduction. Clin Ther 2007; 29: 763 777 .

[27] Santos FL, Esteves SS, da Costa Pereira A, et al. Systematic review and meta-analysis of clinical trials of the effects of low carbohydrate diets on cardiovascular risk factors. Obes Rev 2012; 13: 1048-1066.

[28] Hutton B, Fergusson D. Changes in body weight and serum lipid profile in obese patients treated with orlistat in addition to a hypocaloric diet: a systematic review of randomized clinical trials. Am J Clin Nutr 2004; 80: 1461-1468.

[29] Bravata D, Sanders L, Huang J, et al. Efficacy and safety of low-carbohydrate diets: a systematic review. Jamahttp://jamanetwork.com/journals/jama/fullarticle/1963 51 (2003, accessed 23 February 2017).

[30] Fock KM, Khoo J. Diet and exercise in management of obesity and overweight. J Gastroenterol Hepatol 2013; 28 Suppl 4: 59-63.

[31] Jensen MD, Ryan DH, Apovian CM, et al. 2013 AHA/ACC/TOS Guideline for the Management of Overweight and Obesity in Adults. Circulation. 